\title{
Shangaan patients and traditional healers management strategies of hypertension in Limpopo Province
}

\author{
PR Risenga, MA Cur \\ University of Venda for Science and Technology \\ A Botha, D Cur \\ University of Pretoria \\ JE Tjallinks, MA Cur \\ University of South Africa
}

\section{Correspondence address:}

Edward Rankhumise

MTech (Business Administration)

Tshwane University of Technology

Private Bag X31

Rosslyn, 0200

Tel.: (012) 521066

Fax:(012) 5210823

E-mail : rankhumiseem(àtut.ac.za

\begin{abstract}
Curationis 30(1): 77-84
The study explored the cultural care beliefs, values and attitudes of Shangaans patients' and traditional healers' management strategies of hypertension in the Limpopo Province.

The study aimed to describe the cultural values, beliefs and practices including taboos, rituals and religion within the world-view of the Shangaans. The study was undertaken in the Mopani region of the Greater Giyani area, with the purpose of recommending improvements to patient care in this area.

Data collection was done by conducting focus groups and individual interviews. The following themes emerged

- Hypertension

- The traditional healer: the instrumental role

- Traditional medicine versus Western medicine

- Magico-religious healings

- Cultural beliefs of Shangaans and hypertension

- Experiences of hypertensive patients with regard to traditional healers and hypertension
\end{abstract}

\section{Introduction}

This study focused $\mathrm{n}$ the cultural care beliefs, values and attitudes relating to hypertension among Shangaans in the Limpopo Province of South Africa. Nurses should render competent and culturally sensitive nursing care to all clients. Newman (1990:199) stressed that nurses with different cultural insight and a deep appreciation of human life and values would be able to render culturally appropriate, individualised care.

Nurses should be knowledgeable about human diseases and the beliefs and customs of different cultural groups concerning these diseases. In addition, nurses should show respect, develop a non-judgemental knowledge about other cultures, and understand how culture influences health and illness in different ethnic groups.

A major challenge facing the nursing profession is to provide culturally sensitive care and possibly to move away from a narrowly defined biomedical model of care. This study focused on hypertension amongst the Shangaans in South Africa. 


\section{Background to the problem}

Hypertension is a serious health problem because the treatment poses complex nursing and medical problems.

Because of the complexity of the problem of hypertension amongst the Shangaans, nurses need to increase and direct their efforts towards prevention and towards helping clients to implement lifestyle alterations that will assist in controlling hypertension (Boyle\& Andrew 1995:238; Nkhensani Hospital 1996: 53).

The hospital in the Greater Giyani Lowveld region of the Limpopo Province has three health centres and the clinics as well as mobile clinics, attached to it. The nearest clinic is about 10 kilometres, and the furthest are about 55 kilometres from the hospital. Hypertensive adults between the ages 40 and 65 are admitted to the hospital monthly due to collapse, stroke and high blood pressure. Other patients die before they reach the hospital.

These problems may be due to the difficulties patients experience in complying with long-term drug regimens and lifestyle changes that require major behavioural adaptation. Often, these behavioural changes may present challenges to cultural values, patterns and traditions such as dietary habits, daily activities and lifestyle.

The hospital in Greater Giyani has one adult medical ward with 70 beds to accommodate all medical patients including hypertensive patients. This creates an accommodation problem for the hospital, and leads to some patients sleeping on the floor. Such a situation is not conducive to proper recovery, and rendering of nursing care becomes impossible. The hospital uses Open days to examine hypertensive patients and to distribute treatments. Open days means patients with different problems such hypertension are allowed to go to the hospital any day of the week and this promotes accessibility of Health services to all the clients.

A common view of hypertension amongst the Shangaans in the Limpopo Province was expressed on the radio (Munghana Lonene on 9 June 1997 at 19h00). Stanley Zitha, the SABC presenter, interviewed one patient suffering from hypertension. The patient said hypertension was caused by high salt intake and she was not sure of the treatment or of the effects of hypertension and its complications.

\section{Problem statement}

During 1992-1993, the researcher worked as a professional nurse in different $\mathrm{PHC}$ clinics in the Greater Giyani area. Many Shangaan patients were admitted to hospital due to complications of hypertension, specifically "strokes" or cardiovascular incidents. At one of these clinics three hypertensive women were brought to the clinic with "strokes".

This motivated the researcher to do research on Shangaans with hypertension. Treatment facilities for hypertensive disorders are available, but there is nevertheless proof of a high mortality rate amongst patients diagnosed as hypertensive.

Such incidents indicated that a possible cultural gap between the medical staff at the hospital and the Shangaan patients could exist. The question which arose was:

"What are the cultural care beliefs, values, and attitudes in relation to hypertension of Shangaans of the Limpopo Province?"

\section{Research objectives}

The objectives of this study were:

- to explore cultural values, beliefs and practices such as taboos, rituals, socio-cultural religion within the worldview health, disease specifically in relation to hypertension of selected shangaans in the Mopani region in greater giyani in the Limpopo province.

- to make recommendations for health education in order to make it possible to provide culturally sensitive patient care.

\section{Research methodology Research design}

A qualitative research approach was used to generate knowledge concerned with meaning and discovery (Burns \& Grove 1993: 28) about hypertension and Shangaans.

An exploratory, descriptive and contextual qualitative research design was used (Mouton \& Marais 1998: 102103; Mouton: 1996:133). This design could provide data about the present and about people's thoughts, actions, anticipations and plans in their natural environment, maintaining the emphasis on the natural world of humans (Polit \& Hungler 1991: 178).

The qualitative definition applied for this research is the one presented below by Uys \& Basson (1996) because the study wanted to discover meanings and describe the cultural care beliefs, values and attitudes of Shangaans in relation to hypertension. Qualitative research is a way to gain insight through discovering meanings. Uys and Basson (1996:5) define qualitative research as the approach or methodology, which concentrates on human actions. A literature review revealed numerous ways of interpreting qualitative research.

According to Mouton and Marais ( 1990; 43) the aim of exploratory research is to explore a relatively unknown research area in order to gain new insights into the phenomena under study, rather than collecting accurate and replicable data.

During this study, the researcher explored the literature and the natural settings of Shangaans and their experiences with hypertension as a disease as well as their cultural care beliefs, values, and attitudes in relation to hypertension in Mopani district Greater Giyani. This was done in order to extend the theoretical knowledge available about the Shangaans and cultural practices.

During descriptive research, emphasis is placed on an in-depth description of a specific individual, situation, groups, interaction or social object (Mouton \& Marais; 1990:43-Mouton; 1996:133).

During this study the cultural beliefs, values, and attitudes of Shangaans in relation to hypertension was described. This led to a more understanding of Shangaans and their culture in relation to hypertension amongst them as an ethnic group.

According to Mouton (1998:50) the aim of contextual research is to give an extensive and in-depth description of the phenomena, event or group within the context of the unique setting of the domain phenomenon.

This study is contextually bound to the unique time, space and value context of the specific villages cultural care beliefs, values, attitudes of Shangaans in relation 
to hypertension where the study was conducted. It is site-specific and can only be an educational setting in those villages namely Hlaneki, Dzumeri, Nkuri and Giyani. Thus, these findings are only valid within the context of the specific communities in Greater Giyani in Mopani District the Limpopo Province.

\section{Population and Sample}

The populations for the study were hypertensive patients and traditional healers who were Shangaans. Polit and Hungler (1995:230) described an accessible population as those people who conform to the eligibility criteria. For this study the accessible population comprised that portion of the target population that the researcher could access, hypertensive patients and traditional healers in Mopani Region in Limpopo Province.

A sample refers to the subset of units or elements/humans that comprise of the population (Polit \& Hungler 1995: 230) .In this study the elements were named informants. Field and Morse $(1990 ; 138)$ described an informant as the one from whom the majority of information is obtained.

A nonprobability purposive sampling design was used to select informants. The informants who met the eligibility criteria as hypertensive patients making use of the care provided by traditional healers, and the traditional healers concerned with providing such care were selected for the study. The informants were contacted and agreed to participate in the research, with informed consent (Bless\& Higson-Smith; 1995:95; Streubert \& Carpenter; 1999: 58; Treece \& Treece; 1986: 127). The size of the sample included 30 hypertensive patients and 15 traditional healers.

\section{Data collection}

Traditional healers who met the sampling criteria were interviewed individually and hypertensive patients participated in focus group interviews at various points in comfortable environments (Streubert \& Carpenter; 1999: 23; Wood; 1988:326). The researcher encouraged the informants to speak in Tsonga as they would be speaking to each other in their cultural context in order to be able to present the true reflections of the data without distorting the information, translations were done by an expert in Tsonga and English and after translations information was presented to informants in order to confirm if the information still carries the presented data during the initial interviews for their confirmation. Bracketing, intuiting and reflexivity were continuously reviewed to prevent bias of the researcher (Polit \& Hungler; 1995: 636; Wilson: 1993:179).

Bracketing is the process of identifying and setting aside any preconceived beliefs and opinions one might have about a phenomenon under investigation it involves laying aside what is known. the researcher laid aside information known related to hypertension (Brink; 1999:120).

Intuiting occurs when the researcher tries to develop an awareness of the lived experiences without forcing prior expectations or knowledge in the process. The researcher reviewed the data again and again until there was common understanding. (Brink; 1999:120).

Reflective remarks reflected any thoughts, feelings, ideas, or insights the researcher wished to make, the researcher explored personal feelings and experiences that may influence the study and integrated that understanding into the study. (Burns \& Grove; 1993: 567).

\section{Data analysis}

Data analysis was started immediately after data collection using content analysis and Tesch's stages of data analysis. Bracketing. intuiting and reflexivity were used to exclude preconceptions of the phenomenon in order to enter the world of the informants with open mind.

\section{Documentation of raw data}

Field notes from different interviews with informants were partitioned into individual documents. All data were documented and transcribed verbatim from the tapes. Observations made from all informants during interviews were documented. The pages were numbered from the first interview in the first village to the last interview and this data put together according to the file-card system (Creswell J 1994:154-155)

Data was then compared against the taperecorded and documented ones for accuracy. The data was then coded by the researcher using the mnemonic codes to assist in remembering the category. Data coding was constructed from the data. Data that belonged to one group was assembled in one place to assist further reading. Themes and categories were also formulated according to the data themselves with the assistance of data obtained during literature review.

\section{Measures for ensuring trustworthiness}

The goal of qualitative research is to accurately represent the informants' experiences. Guba (1981) and Lincoln and Guba (1984) suggested four criteria to support trustworthiness, these criteria are credibility, dependability, confirmability and transferability. The criteria and strategies were used in the study to ensure trustworthiness.

Truth value establishes how confident the researcher is with the truth of the findings based on the research design, informants and context. In qualitative studies truth is assessed by how well threats to the internal validity of the study and the instrument as a measure of the phenomenon under study, have been managed. Internal validity is supported when changes in the dependent variable are accounted for by changes in the independent variable, that is, when the design minimises the effects of competing confounding variable by control or randomisation. Lincoln and Guba (Guba; 1981:80) termed this credibility (Krefting; 1991:215).

For this study credibility was attained as the information related to cultural care beliefs, values and attitudes of Shangaans in relation to hypertension was discovered through the use of interviews and that was true, human experience as lived by informants which included both the traditional healers and hypertension patients who are Shangaans in those areas namely Hlaneki, Dzumeri, Nkuri and Giyani. The culture care beliefs, values and attitudes of Shangaans in relation to hypertension was not defined by the researcher but by the informants themselves. Informants including both traditional healers and hypertensive patients were given a chance to describe their experiences while the researcher listened and recorded all the information said by the informants.

Applicability refers to the degree to which the findings can be applied to other contexts and settings or with other 
groups. It is the ability to generalise from the findings to larger populations. In qualitative studies applicability refers to how well the threats to external validity have been managed (Sandelowki; 1986:30). Payton as cited in( Krefting; 1991: 216) defined external validity as the ability to generalise from the study sample to the larger population and hence, the importance of sampling technique is establishing external validity.

Sandelowki (1986:32) suggests that generalisation is relevant in many qualitative research studies. These studies are undertaken in naturalistic settings with few controlling variables. Each situation is unique and thus less amenable to generalisation. Generalisation is an illusion as every research situation is made up of particular informants. Applicability is irrelevant to qualitative research as its purpose is to describe a particular phenomenon or experience, not to generalise to others.

Guba (1981:81) refers to fittingness or transferability as the criterion against which applicability of qualitative data is assessed. Qualitative research meets this criterion when the findings fit into contexts outside the study situation that are determined by the degree of similarity of goodness of fit between the two contexts.

Lincoln and Guba (1985) as cited in (Krefting; 1991: 216) state that transferability is more the responsibility of the person wanting to transfer the findings to another situation or population, than the researcher of the original study. As long as the original researcher presents sufficient descriptive data to allow comparison, she has addressed the problem of applicability. The study addressed the issue of applicability in this study because all sufficient descriptive data related to cultural care beliefs, values and attitudes of Shangaans in relation to hypertension was clearly presented in chapter four which allows for comparison of the informants with other cultural groups namely the Venda speaking, Sotho, Zulu as well as Tsonga / Shangaans from other areas outside the villages used for this study.

Consistency refers to whether the findings would be consistent if the study was replicated with the same informants or in a similar context (Guba; 1981: 80).In contrast to the relatively controlled, structured experimental environment, the qualitative settings may be complicated by extraneous and unexpected variables leading to an unstructured and often spontaneous settings (Duffy: 1985:230). The instruments that are assessed for consistency in qualitative research are the researcher and the informants, both of whom vary greatly within the project.

Qualitative research expresses the uniqueness of the human situation, so that variation in experience, rather than identical repetition is sought (Sandelowki; 1986:33). Thus, variability is expected in qualitative research and consistency is defined in terms of dependability. Guba (1981:81) defines dependability as track able variability, that is, variability that can be ascribed to identified sources (Krefting; 1991:216). Since well consistency is defined in terms of dependability, this study ensured dependability of the study by providing a complete description of methodology, coding of data by the researcher together with independent coders. Therefore the study followed requirement to ensure consistency of the data obtained.

Neutrality refers to the degrec to which the findings are a function solely of the informants and conditions of the research and not of biases, motivations and perspectives, that is, freedom from bias in the research procedures and results (Krefting; 1991:216)

During qualitative studies, the researcher trics to increase the worth of the findings by decreasing the distance between the researcher and informants, for example, by prolonged contact with informants or lengthy periods of observation. Lincoln and Guba (1985) as cited in (Krefting; 1991:217) shifted the emphasis of neutrality in qualitative research from the researcher to the data, so that neutrality and not investigator neutrality was considered. They suggest that conformability be the criterion of neutrality. This is achieved when truth value and applicability are established (Guba; 1981:82). For this study neutrality was achieved because truth value and applicability were clearly explained and achieved by describing the informants real experiences presented by informants themselves and this allows for applicability of information to other areas. It was also achicved by doing conformability audit where data was given to independent coders from one of the University in the Limpopo Province for coding together with the researcher.

\section{Ethical consideration}

Ethical considerations were adhered to, prevent ethical problems. Permission from Greater Giyani Municipality was obtained (annexure). Rights of informants were respected through the study. Informed consent, confidentiality, anonymity and risk / benefit ratio were maintained throughout the study.

\section{Informed consent}

This principle was addressed by giving informants full information about the purpose of the study, its significance, and data collection procedures before data was collected. This principle received priority consideration since informants were patients and they might have perceived the researcher as having authority over them. They were informed that participation was strictly voluntary and a consent form was signed by informants (see annexure D for consent form).

\section{Confidentiality and anonymity}

Confidentiality in this was not easy since precise quotations from transcripts had to be included in the collection of data. Informants were reassured that information gained would not be linked to their names. Anonymity was provided by protection of the informant's identities. They were assured that their names would not be used after data analysis had been completed. Anonymity was maintained during publication by omitting identifiable data, such as names on transcripts.

\section{Risk/benefit ratio}

This principle means that the degree of risk taken by those participating should never exceed the potential humanitarian benefits of the knowledge to be gained. Qualitative research is considered noninvasive, depending on the topic under study. This study focused on a significant topic that had a potential to improve care of hypertensive patients.

\section{Presentation of findings}

Themes and categories were also formulated according to the data themselves with the assistance of data obtained during literature review. 
Formulated themes for data obtained during individual interviews with traditional healers are as follows.

- $\quad$ Theme 1 Hypertension
- The instrumental role
- Theme 3 Traditional Medicine
versus Western Medicine
- Theme 4 Magico-religious
Theme 5 Cultural beliefs of
Shangaans and hypertension
Theme $6:$ Experiences of
hypertensive patients with
regard to traditional healers and
hypertension

\section{Theme one: Hypertension}

According to informants discussions four categories emerged from the data related to hypertension. Each category was discussed separately.

\section{Category one: Synonyms for Hypertension}

According to informants own views and perceptions no subcategory emerged as only three names are associated with hypertension according to the Tsonga / Shangaans culture namely Ngatileyikulu, N'ombe or High blood. All the informants presented these words only.

\section{Category 2: Signs and symptoms}

The results revealed that specific signs and symptoms were related to high blood. According to their views signs and symptoms discussed included continuous headaches, dizziness, painful body, swelling, failure to see properly, protruding blood vessels, tiredness, nose bleeding, collapse and faint.

Results revealed that Shangaans believe that all symptoms have cultural meaning. As they experience symptoms, they interpret them and react in ways that are related with their cultural norms.

\section{Category 3: Remedies}

Remedies discussed by informants included a variety of activities moving from indications, prescriptions, positive remedies and negative remedies as well as the preparation methods, related to individual remedies from which individual themes emerged.

\section{Subcategory 2: Remedies indications/prescription}

Informants presented different prescriptions for treatment of Ngatileyikulu/N'ombe as well as correct times for utilization of such prescribed herbal medicine the signs and symptoms presented by hypertensive patients were the guidelines for the prescription hence each patient would be treated according to the signs and symptoms on admission to the traditional healer as follows:

- $\quad$ Liquid herbal medicines - ls used for tiredness and excessive sweating as experienced by hypertensive patients

- Dry roots of herbal medicine for headache

- $\quad$ Burned moulds and herbal medicine - to loosen blood which is believed to be high and thick related to hypertension.

- Baso-For dizziness

- Steam inhalation - for washing blood

- Grinded herbal medicine mixed with fat - for rubbing affected parts in stroke

\section{Subcategory 3: Remedies positive}

Informants described positive remedies related to the herbal medicine prescribed to yield positive result as it is given according to specific indications. The following remedy was reported by traditional healers

- Ku Lumeka-Scarification and application of herbal medicine very good for High blood.

\section{Subcategory 4: Remedies Negative}

Informants illustrated that there are specific remedies that are not supposed to be used for specific people due to their conditions in order to avoid complications.

- Do not do phungula (steam inhalation) to people with stroke because it is believed that the method will lead to collapse of the people exposed to the treatment method.

\section{Subcategory 5: Remedies Preparation}

Informants gave clear methods for preparation in order to yield positive results. Methods were summarized and presented Informant's results revealed that the Shangaan/Tsonga people have knowledge with regard to remedies to be utilized for hypertension.

\section{Category 6: Treatment Fe edback}

From the category, there are no themes that emerged. Some informants explained that after the sick person has taken the medication, they must go back to traditional healers to explain the results of herbal medicine in use.

Theme two: The traditional healer: The instrumental role

Informants described specific aspects related to diagnostic procedures followed by Traditional healers during the treatment process. This explanation was coupled by the presence of confidence for the procedures done. Two categories emerged from the data and each category was discussed separately to represent data units in each category.

\section{Category 5: Sources for diagnostic assistance}

- Throwing of bones and procedures-payment, untie the bag, client blow air onto the bones then throw bones down.

- Interpretations of bones - look at position adopted by the bones and assign meaning, then diagnose the person.

- Throw bones to ask the ancestors the herbal medicine relevant for the disease.

- Diagnosis and dreams -

Ancestors can come to you in a dream to tell you events which are going to happen the following day indicating the specific person, diagnosis, and treatment to be implemented.

\section{Theme three: Traditional} medicine versus western medicine

Subcategory 1: Attitudes towards Western Medicine

Informants explained that Western medicine must be utilised for complications such as stroke because the traditional healers do not have the necessary facilities to cater for complications.

\section{Subcategory 2: Feelings about preparation of Western medicine towards hypertension}

Other informants blamed Western medicine for prolonged use of medicine for treatment of hypertension.

Subcategory 3: Recommendations by traditional healers

- Inviting traditional healers to 
assist in treatment of chronic conditions such as hypertension.

Western medicine for treatment of complications only such as stroke.

\section{Theme four: Magicoreligious}

Informants displayed the presence of the ancestors throughout their lives whether; they are healthy or sick and stressed that for things to go normally proper communication must be maintained throughout. The presence of ancestors represents the Magicoreligious aspects of Shangaans according to informants' views

\section{Category 8: Relationship with Ancestors}

Informants in this study revealed that there must always be peace between them and the ancestors.

They stressed that threatening the relationship or causing ancestors to be angry might bring ill health. To resolve that problem, a ceremonial sacrifice should be done where a white goat/ chicken is slaughtered and, people specifically relatives are invited to come and enjoy the ceremony and to eat the slaughtered animal.

They believed that good relationship with ancestors is going to promote someone's health and if he is ill, the traditional healer will provide the treatment for different diseases such as hypertension. To maintain, the relationship there must be continuous communication between ancestors and people through the process of libation. Breach of taboos or talking about confidential information to strangers will make ancestors to be angry and therefore it must be avoided

\section{Theme five: Cultural beliefs of shangaans and hypertension}

From the discussions with informants, certain cultural beliefs and values with regard to hypertension as a disease were outlined.

\section{Beliefs}

Subcategory 1: Causes of hypertension Informants explained that there are several aspects responsible for causing hypertension though others seemed to be beyond their control.

Excessive intake of salt and fat, anger of ancestors and it seem to run in families and the cause is not clearly known.

\section{Subcategory 2: Report to ancestors}

The informants stressed that all the events occurring in the family must be reported to ancestors, if someone is ill, ancestors must be informed.

\section{Subcategory 3: Medication}

Informants discussed that there is no need for a person to live on life-long medication because hypertension is curable.

\section{Subcategory 4: Cultural care of a hypertensive patient.}

Informants demonstrated that there are specific people such as the elderly who are allowed to look after this person when ill because they are no longer exposed to issues which make them to be HOT.

\section{Subcategory 5: Prognosis}

Informants illustrated that people with hypertension are treated successfully without any need for prolonged drugs.

\section{Theme six: Experiences of hypertensive patients with regard to traditional healers and hypertension}

The informants described many aspects under their experiences, from the descriptions the following category and subcategories emerged.

\section{Category 1: Experiences sign and symptoms}

Informants tried to indicate the intensity of the headache experienced on first attack which was followed by dizziness as the haunting problems.

\section{Category 2: Experiences and causes} Informants explained that there are many aspects responsible for hypertension.

\section{Category 3: Experiences with Sources for Diagnostic Assistance}

Informants reported that they are expected to pay specific amount before the diagnosis is done.

\section{Category 4: Experiences with Remedies}

Informants indicated fear, pain and agony related to some other methods utilized as remedies, such as Ku lumeka and steam inhalation

\section{Category 5: Prognosis}

Some informants reported they were completely healed after the visit to the traditional healer.

\section{Recommendations}

Based on the conclusions deduced from the findings of this research, recommendations were made. Changing of attitudes by health professionals such as development of positive relationship towards the traditional healers, close collaboration between Western medicine and traditional healers, workshops with the traditional healers and further research.

\section{Development of positive relationship towards the traditional healers}

Health professionals must learn and try to develop good attitudes towards the Traditional Healers. Once the relationship is established, it will promote good working relationship. This will promote reliance of both systems for something and in return; the medical systems will rely on Traditional Healers for something. This is very important because both traditional healers and medical practitioners have one common goal, to help the patient get well. Both they are patient orientated. Once the Traditional Healers became satisfied with relationship and negotiations that they are being consulted, as equals in their own right mistrust will be dispelled.

The nursing profession need to avoid stereotyping of the herbal medicine, but rather must take time to learn something about the practice of the Traditional Healers in order to be able to view it in cultural context

Development of good attitudes towards the Traditional Healers will drew them nearer to health professionals and therefore close collaboration will be easy.

\section{Close collaboration between western medicine and the $\mathrm{TH}$}

Health professionals should develop positive attitudes towards the Traditional Healers. Both traditional healers and medical practitioners have one common goal, to help the patients get better. Once the Traditional Healers became satisfied with relationship and negotiations that they are being consulted may be care for hypertensive patients might improve.

A study conducted by Oberholzer as cited in Gumede (1990: 230) through community-orientated programme, consulting the Dingaka council (a local 


\begin{tabular}{|c|c|c|c|c|}
\hline \multicolumn{2}{|c|}{ THEME 1} & \multicolumn{2}{|c|}{ CATEGORIES } & SUBCATEGORIES \\
\hline 1. & Hypertension & 1.1 & Synonyms for hypertension & $\begin{array}{ll}\cdot & \text { N'ombe } \\
- & \text { Ngatileyikulu } \\
- & \text { High blood }\end{array}$ \\
\hline & & 1.2 & Signs and symptoms & $\begin{array}{ll}\cdot & \text { Headache } \\
\cdot & \text { Dizziness } \\
\text { - } & \text { Nose Bleeding } \\
\text { - } & \text { Tiredness }\end{array}$ \\
\hline & & & Remedies & $\begin{array}{ll}- & \text { Indications } \\
- & \text { Preparation } \\
- & \text { Positive effects } \\
- & \text { Negative effects }\end{array}$ \\
\hline \multirow[t]{2}{*}{2.} & $\begin{array}{l}\text { The traditional healer: the } \\
\text { instrumental role }\end{array}$ & 2.1 & $\begin{array}{l}\text { Sources for diagnostic } \\
\text { assistance }\end{array}$ & $\begin{array}{ll}- & \text { Procedures for throwing bones } \\
\text { - } & \text { Throwing of bones } \\
\text { - } & \text { Interpretation of bones } \\
\text { - } & \text { Bones and ancestors } \\
& \text { Diagnosis and dreams }\end{array}$ \\
\hline & & 2.2 & $\begin{array}{l}\text { Traditional healer self } \\
\text { confidence }\end{array}$ & $\begin{array}{ll}- & \text { Faith in oneself } \\
\text { - } & \text { Throwing of bones } \\
\text { - } & \text { Trust in herbal medicine }\end{array}$ \\
\hline 3. & $\begin{array}{l}\text { Traditional medicine versus } \\
\text { Western medicine }\end{array}$ & 3.1 & $\begin{array}{l}\text { Attitudes towards western } \\
\text { medicine }\end{array}$ & $\begin{array}{ll}- & \text { Traditional healers attitudes } \\
\text { - } & \text { Feelings about Western } \\
& \text { medicine for hypertension }\end{array}$ \\
\hline 4 & Magico-religious healing & 4.1 & Relationship with ancestors & $\begin{array}{ll}\text { - } & \text { Approval of ancestors } \\
\text { - } & \text { Communication with ancestors }\end{array}$ \\
\hline 5 & $\begin{array}{l}\text { Cultural beliefs of Shangaans } \\
\text { and hypertension }\end{array}$ & 5.1 & Beliefs & $\begin{array}{ll}\text { - } & \text { Causes of hypertension } \\
\text { - } & \text { Reporting to ancestors } \\
\text { - } & \text { Medication } \\
& \text { Cultural care of a hypertensive } \\
\text { - } & \text { patient } \\
\end{array}$ \\
\hline 6 & $\begin{array}{l}\text { Experiences of hypertensive } \\
\text { patients with regard to } \\
\text { traditional healers and } \\
\text { hypertension }\end{array}$ & 6.1 & $\begin{array}{l}\text { Experiences of hypertensive } \\
\text { patients }\end{array}$ & $\begin{array}{ll}\text { - } & \text { Experiences of signs and } \\
\text { symptoms } \\
\text { - } & \text { Patients beliefs about causes } \\
\text { - } & \text { Experiences of diagnostic } \\
\text { methods }\end{array}$ \\
\hline
\end{tabular}

TH's council) made important observations such as: the Traditional Healers felt the western healers / doctors wanted to subjugate them \& manipulate them into working under the supervision of medical practitioners. The Traditional Healers wanted to implement a liaison. The nursing profession need to avoid stereotyping of the talk medicine, but rather must take time to learn anything about the practice of the TH in order to be able to view it in cultural context (Leininger; 1991: 39)

Western healers need to come out of the 
temples of learning on the hilltops and come dawn to the valleys where the people are (Gumede; 1990: 235). The above statement clearly support and promote the issue of collaboration to establish health systems. The issue is no longer one formal or informal health care; both western and traditional healing should be welcome (Gumede; 1990: 235). The World Health Organisation in Gumede (1990: 235-237) set a target to achieve health for all by the year 2000 , and it was stated clearly that this cannot be done on the western model alone, other alternatives will have to be found. The traditional healers according to their discussions in this study seemed to be willing and ready to talk. Health professionals must make it their task to the communities to address the problem and to ensure a system of proper collaboration of the two systems (western and generic).

\section{Workshop with traditional healers}

Health professionals including nurses, medical practitioners must organize workshops for the traditional healers in order to share more information in relation to hypertension.

During the workshop, the Traditional Healers need to be given on opportunity to present their point of view. Thorough assessment of their information must be done by the health personnel. At the end, the three modes of Leininger's sunrise model must be adopted. Good practices which help the Shangaan to retain and preserve relevant culture values \& maintain their well being and help them recover from illness related to hypertension will be maintained and preserved.

The harmful practices such as the use of razor blade during the treatment process to cut the skin to allow blood out in a patient with hypertension negotiations can be made in order to help them to restructure while respecting their cultural values, beliefs in order to assist them to cope with the disease.

\section{Strenghs of the study}

Strengths of the study include the following:

- The qualitative approach and unstructured interviews allowed the informants to speak for themselves.

- This was not a laboratoryoriented study, but was implemented in the field.

- The researcher was conversant with the situation and was more accessible to the traditional healers due to familiarity with the context in which the study was conducted.

The findings have made a contribution to the knowledge of management of hypertension amongst the Shangaans. The findings increase the knowledge of the phenomenon and establish an understanding of Shangaan culture and hypertension

\section{Limitations of the study}

Limitations for this study include the possibility of researcher's bias and data collection as well as data analysis. The researcher's bias involved in this research might include aspects such as the researcher's knowledge of hypertension which indicate that the researcher came into the conduction of the study with the knowledge though bracketing was done. Certain limitations were identified in the study namely: participant effect, and data collection and analysis. This is a contextual study and the findings of this study cannot be generalised.

\section{Conclusions}

Cultural care beliefs; values and attitudes are available amongst the Shangaans and other members of different cultural groups.

Shangaans have their own perceptions of high blood / Ngatileyikulu / hypertension and this is influenced by the presence of their own world views, cultural perceptions as well as their experiences in their own culture.

Failure to recognise these beliefs, values and attitudes by nurses and other health personnel who are rendering health care might lead to many complications such as failure of Shangaans who are hypertensives to cope with the chronicity of their condition and failure to adjust to life style changes brought by the disease.

Delivery of culturally competent health care requires that nurses develop positive attitudes towards people from different cultural backgrounds. Nurses need to acquire the knowledge available amongst the different groups of South African population from different cultural background which influence the way people react to health and illness.

\section{Bibliography}

BLESS, C \& HIGSON-SMITH, C 1995.

Fundamental of social research methods. $2^{\text {nd }}$ edition. Cape Town: Juta.

BOYLE, JS \& ANDREWS, MM 1995.

Transcultural concepts in nursing care. Philadelphia: Lippincott.

BURNS, N \& GROVE, SK 1993. The practice of nursing research. Philadelphia: Saunders.

FIELD, PA \& MORSE, JM 1990. Nursing research: the application of qualitative approaches. Berlin: Springer

GUBA, EG 1981. Understanding and doing naturalistic inquiry. Beverly Hills, Califomia: Sage.

GUMEDE, MV 1990. Traditional healers: a medical doctor's perspective. Johannesburg: Blackshows.

LEININGER, MM 1991. Cultural care diversity and universality: a theory of nursing. New York: National League for Nursing Press.

MOUTON, J 1996. Understanding social research. Pretoria: Van Schaik

MOUTON, J \& MARAIS, HC 1998. Basic concepts in the methodology of social science. Pretoria: Van Schaik.

NEWMAN, M 1990. Theory development in nursing. Philadelphia: Davis.

NKHENSANI HOSPITAL 1996. Hypertension monthly statistics. Giyani.

POLIT, DF \& HUNGLER, BP 1991. Nursing research principles and methods. Philadelphia: Lippincott.

POLIT, DF \& HUNGLER, BP 1995. Essentials of nursing research. Philadelphia: Lippincott.

STREUBERT, HJ \& CARPENTER, DR 1999. Qualitative research in nursing advances: the humanistic imperative. Philadelphia: Lippincott.

WILSON, AG 1993. Designing an integrated model. London : Routledge 